\title{
Atmospheric-Pressure Solution-Cathode Glow Discharge: A Versatile Ion Source for Atomic and Molecular Mass Spectrometry
}

\author{
Authors: Andrew J. Schwartz, ${ }^{1}$ Kelsey L. Williams, ${ }^{2}$ Gary M. Hieftje ${ }^{1}$ and Jacob T. Shelley, ${ }^{2,3} *$
}

\begin{abstract}
An atmospheric-pressure solution-cathode glow discharge (SCGD) has been evaluated as an ion source for atomic, molecular, and ambient desorption/ionization mass spectrometry. The SCGD consists of a direct-current plasma, supported in the ambient air in the absence of gas flows, and sustained upon the surface of a flowing liquid cathode. Analytes introduced in the flowing liquid, as an ambient gas, or as a solid held near the plasma are vaporized and ionized by interactions within or near the discharge. Introduction of acidic solutions containing metal salts produced bare elemental ions as well as $\mathrm{H}_{2} \mathrm{O}, \mathrm{OH}^{-}$ and $\mathrm{NO}_{3}{ }^{-}$adducts. Detection limits for these elemental species ranged from 0.1 to $4 \mathrm{ppb}$, working curves spanned more than 4 orders of linear dynamic range, and precision varied between 5 and 16\% relative standard deviation. Small organic molecules were also efficiently ionized from solution, and both the intact molecular ion and fragments were observed in the resulting SCGD mass spectra. Fragmentation of molecular species was found to be tunable; high discharge currents led to harder ionization, while low discharge currents produced stronger molecular-ion signals. Ambient gases and solids, desorbed by the plasma from a glass probe, were also readily ionized by the SCGD. Indeed, strong analyte signals were obtained from solid samples placed at least $2 \mathrm{~cm}$ from the plasma. These findings indicate that the SCGD might be useful also for ambient desorption/ionization mass spectrometry. Combined with earlier results that showed the SCGD is useful for ionization of labile biomolecules, the results here indicate that the SCGD is a highly versatile ion source capable of providing both elemental and molecular mass-spectral information.
\end{abstract}

\section{Introduction}

Originally developed as alternative atomization and excitation sources for atomic emission spectrometry (AES), solution-electrode glow discharges (SEGDs) have attracted the attention of the spectrochemistry community because of their advantages over conventional plasmas for AES, such as the inductively coupled plasma (ICP). Comprised of low-power, atmospheric-pressure glow discharges where one or both electrodes has been replaced with flowing electrolyte solutions, SEGDs boast simple, inexpensive designs that overcome drawbacks associated with high-power plasmas often used for elemental analysis. Unlike the ICP, which is bulky, requires $1-2 \mathrm{~kW}$ radiofrequency power and high flows of compressed gas $\left(\geq 15 \mathrm{~L} \mathrm{~min}^{-1} \mathrm{Ar}\right)$, SEGDs require little or no compressed or flowing gases, utilize low $(<100 \mathrm{~W})$ direct-current power, and desolvate/excite analytes directly from a sample solution, 
resulting in a rapid temporal response and obviating the need for sample-solution nebulizers or spray chambers.

Though many SEGD designs have been reported in the literature, two geometries have received the most attention. The first, based on the format reported in 1993 by Cserfalvi et al. [1], is known as the electrolyte as cathode discharge (ELCAD) or the solution-cathode glow discharge (SCGD) [2-8]. Sources of the SCGD geometry are comprised of an electrical discharge (approximately 1-3 $\mathrm{mm}^{3}$ volume) sustained between an upper metal anode rod and a flowing cathodic solution provided through a glass capillary. Overflow of the solution from the capillary tip is used to create an electrical connection to ground potential, either by direct contact with a grounded electrode or by drainage into a solution reservoir held at ground. The SCGD has been shown to exhibit impressive analytical performance for AES; detection limits for metals in acidic solutions range from 0.06 to $22 \mathrm{ppb}$ [9], comparable to radially viewed ICP-AES. Furthermore, this design is advantageous in that it is typically operated at low-tomoderate powers $(35-80 \mathrm{~W})$ and does not require any compressed or flowing gas; the ambient air serves as the plasma gas. The greatest drawback of the SCGD is that a relatively high solution flow rate (typically $1.0-4.0 \mathrm{~mL} \mathrm{~min}^{-1}$ ) is required to maintain the solution overflow and, thereby, connection to electrical ground. However, flow injection strategies have successfully reduced sample consumption with little or no loss in analytical performance $[10,11]$.

The second dominant class of SEGDs is the design reported by Marcus and Davis in 2001 [12], known as liquid-sampling atmospheric-pressure glow discharge (LS-APGD). In the LS-APGD, solution is provided through a fused-silica capillary sheathed by an electrically grounded, stainless-steel tube. A nickel counter electrode is placed within two millimeters of the capillary, and an electrical discharge 1-2 $\mathrm{mm}^{3}$ in volume is sustained on the liquid. Unlike the SCGD, the LS-APGD utilizes a He or Ar sheath gas provided at a rate of $0.1-1.0 \mathrm{~L} \mathrm{~min}^{-1}$ through the stainless steel tube and is typically operated at lower powers $(5-50 \mathrm{~W})$ [13]. Furthermore, the LS-APGD consumes significantly less solution than does the SCGD, employing flow rates that range from 5 to $500 \mu \mathrm{L} \mathrm{min}^{-1}$. At these low rates of sample consumption, the discharge often operates in a total-consumption mode in which all of the liquid provided through the capillary is vaporized. Analytical performance of the LS-APGD is more modest than that of the SCGD; AES detection limits for 5- $\mu \mathrm{L}$ injections range from 4 to $10 \mathrm{ppm}$ (20-45 ng absolute mass) for most elements $[12,13]$.

Recently, the LS-APGD was investigated as an ion source for mass spectrometry (MS). Despite the low applied power $(<10 \mathrm{~W})$ and limited plasma volume in which desolvation can occur, the LSAPGD efficiently produced bare elemental ions from acidic solutions of aqueous metal salts. Detection limits with LS-APGD-MS (0.02-2.2 ppm) [14, 15] were better than those obtained with AES (4-10 ppm) $[12,13]$, though this sensitivity is poor in comparison to ICP-MS where single or sub-parts-per-trillion detection limits are common [16]. Later studies revealed that changes to the supporting electrolyte composition yielded softer ionization, and production of intact molecular ions $[17,18]$. In particular, when the supporting electrolyte was changed from $1 \mathrm{M} \mathrm{HNO}_{3}$ to a mixture of $70 \%$ methanol and $30 \%$ water, the LS-APGD produced intact molecular ions and fragments from solution, including acetatebound uranyl cations $\left(\mathrm{UO}_{2}{ }^{+}\right)$[17] and a diverse range of organic molecules [18]. Other studies showed that the LS-APGD functioned effectively for ambient desorption/ionization MS of solids and gases [19].

Though extensive work has been performed to characterize the LS-APGD as an ion source, MS applications of the SCGD are only now being pursued. A recent study by Schwartz et al. [20] revealed that the SCGD efficiently produces gas-phase ions of labile biomolecules (peptides) directly from aqueous solution. Ionization and fragmentation of the peptides was controllably tunable through changes in the discharge current. Perhaps counterintuitively, high SCGD currents produced electrospray- 
ionization-like spectra, whereas lower currents resulted in extensive fragmentation, enabling elucidation of the primary structure of the peptides via the series of a-, b-, c-, x-, y- and d-type ions that were produced. Unlike the LS-APGD, no changes to the support solution composition were required to generate the biomolecular ions. Furthermore, the SCGD-MS results were significantly different from those obtained for biomolecular analysis with the LS-APGD [18, 20]. Analysis of myoglobin by LSAPGD yielded an ESI-like charge-state distribution and no fragment ions, apart from loss of the heme group. These differences, combined with earlier observations that suggest the SCGD and LS-APGD function by different desolvation mechanisms (electrospray and thermal, respectively) $[7,12,13]$, suggest the SCGD behaves differently from the LS-APGD and that further investigation of the SCGD as an ion source is merited.

The work presented here constitutes a pilot study to evaluate the analytical potential of the SCGD as an ion source for elemental and small-molecule mass spectrometry. Background and reagent ions formed by the SCGD were evaluated, and a variety of analytes in solution, ranging from elemental to molecular species (both inorganic and organic) were examined. Potential application of the SCGD as an ambient desorption/ionization source for solids and gases was also evaluated. Implications of the results are discussed and preliminary analytical performance is presented.

\section{Experimental}

\section{SCGD Cell, Ion Sampling, and Sample Introduction}

Shown in Figure 1 is a photograph of the SCGD cell and capillary mass-spectrometer interface used for ion sampling. The design of the SCGD cell and interface is identical to that described earlier [20], so only a brief description to indicate differences in parameters will be provided here. In the SCGD of Figure 1, electrolyte solution (either $0.1 \mathrm{M} \mathrm{HNO}_{3}$ or $\mathrm{HCl}$ ) is pumped into the cell through a vertically oriented glass capillary at a rate of $1.7 \mathrm{~mL} \mathrm{~min}^{-1}$. Solution overflow from the tip of the pipet onto an electrically grounded graphite rod positioned 3-mm below the tip of the glass capillary creates an electrical connection between the incoming sample solution and ground. When a high positive potential $(>1000 \mathrm{~V})$ is applied to a tapered tungsten rod, positioned $2.5-\mathrm{mm}$ above the tip of the glass capillary, electrical breakdown results in a glow discharge that is sustained on the surface of the liquid exiting the capillary. Analytes present in the liquid stream, in the ambient atmosphere, or on the tip of a glass probe held near the plasma are vaporized and ionized by interaction with the discharge. Continuous sample introduction was used in all cases where the analyte was introduced within the electrolyte stream. Except where noted in the text, the SCGD was operated in constant-current mode with a current of $70 \mathrm{~mA}$. Conditions utilized for this pilot study were determined from a rough, parametric evaluation of the SCGD ion signals for $\left(\mathrm{UO}_{2}+\mathrm{OH}+\mathrm{H}_{2} \mathrm{O}\right)^{+}$and $\left({ }^{208} \mathrm{~Pb}+\mathrm{H}_{2} \mathrm{O}\right)^{+}$; plots for this evaluation are included within the supplementary material.

Gas-phase analyte ions generated by the SCGD were sampled into the reduced-pressure environment of the mass spectrometer via a customized extended capillary interface heated to $320{ }^{\circ} \mathrm{C}$ by means of the instrument's standard inlet heater, controlled and monitored through the instrument software. No directed gas flows, apart from the vacuum pull of the first stage of the mass spectrometer, were used to direct ions from the SCGD to the capillary inlet. Two mass spectrometers were used: for evaluation of low $\mathrm{m} / \mathrm{z}$ background and reagent ions, a linear ion trap mass spectrometer (LTQ XL, Thermo Scientific, San Jose, CA) was utilized, whereas a high-resolution Orbitrap mass spectrometer (Exactive Plus, Thermo Scientific, Bremen, Germany) was used in all other experiments. The SCGD 
solution capillary was positioned at a distance of 3-mm from the mass spectrometer sampling inlet (cf. Figure 1).

\section{Signal Acquisition and Data Processing}

High-resolution mass spectra were acquired with the Orbitrap mass spectrometer with a resolving-power setting of 140,000 and automatic gain control target values that ranged from $1 \times 10^{6}$ to $5 \times 10^{6}$ ions. The identity of adduct species and fragments detected with SCGD-MS was determined through accurate-mass measurement. Daily mass calibration was performed to ensure mass accuracy better than $1 \mathrm{ppm}$. A lock mass of $\mathrm{m} / \mathrm{z} 371.10124$, due to polysiloxane, was used to compensate for slight scan-to-scan mass drift. In spectra where in-source collision-induced dissociation (CID) was utilized, the applied fragmentation energies ranged from 20 to $40 \mathrm{eV}$.

Except where stated otherwise, mass spectra shown represent the average produced from a 9.4-s integration, corresponding to 10 scans. Limits of detection (LODs) for elemental species were determined from the formula:

$$
L O D=\frac{3 \sigma}{\bar{x}}
$$

where $\sigma$ represents the background noise (in counts, cts) and $\bar{x}$ the sensitivity (cts $\mathrm{ppb}^{-1}$ ) determined from the linear region of the working curve of an element. Since the Orbitrap provides sufficient resolution such that no background signals resulting from isobaric interferences occur and because electronic noise in the mass spectrum is automatically removed by means of a threshold discriminator, a blank solution yields zero ion counts (and thereby zero noise) for all of the elements investigated here. As a result, background noise was instead estimated from a calibration standard near the detection limit; in this case 0.5 or $1 \mathrm{ppb}$. Though this method, commonly employed in MS instruments where there is little-to-no chemical noise [21], enables detection limits to be estimated in the absence of background signal, the "true" limits of detection are likely better than those calculated due to the influence of multiplicative noise sources. Noise was measured as the standard deviation produced from 15 measurements of the elemental signal, where each measurement was a 9.4-s integration (average of 10 mass-spectral scans). Elemental signals were taken as the sum of the peak areas of all isotopes from a given element. Precision (as relative standard deviation, RSD) was determined from the average and standard deviation of the signal produced from five consecutive 9.4-s integrations of the peak area of the analyte at 1000-ppb concentration.

\section{Reagents and Sample Preparation}

All chemicals used for preparation of solution standards and for evaluation of ambient desorption/ionization were of reagent-grade ( $\geq 95 \%$ purity) or better. Molecular standards were prepared by serial dilution of a concentrated $(0.1 \mathrm{M})$ stock solution with $0.1 \mathrm{M} \mathrm{HCl}$ diluent. Elemental standards were prepared by serial dilution in $0.1 \mathrm{M} \mathrm{HNO}_{3}$ of a stock solution (1000 ppm, prepared from the nitrate salts of the respective elements). Here, dilute $\mathrm{HCl}$ was used for molecular solution to prevent potential reactions and/or degradation of organic analytes by action of $\mathrm{HNO}_{3}\left(\mathrm{NO}_{3}{ }^{-}\right.$is a strong oxidizer). Conversely, $\mathrm{HNO}_{3}$ was used for preparation of elemental standards to prevent the formation of insoluble precipitates (such as $\mathrm{AgCl}$ ) and to prevent ligation of the target metal ions by chloride ions. Diluents and SCGD support solutions (either $0.1 \mathrm{M} \mathrm{HCl}$ or $\mathrm{HNO}_{3}$ ) were prepared by dilution of the respective trace- 
metal grade concentrated acid with water of $18.2 \mathrm{M} \Omega \mathrm{cm}$ resistivity, prepared in-house with a mixed-bed ion-exchange deionization unit.

\section{Results and Discussion}

\section{Reagent and Background Ions}

Initial evaluation of SCGD-MS focused on determination of the chemical environment (e.g., background and reagent ions) produced by the source. Figure 2A shows an SCGD mass spectrum obtained during introduction of a blank $0.1 \mathrm{M} \mathrm{HNO}_{3}$ support solution. Under the conditions utilized here, the low-mass range is dominated by lower-order protonated water clusters $\left(\mathrm{H}_{2} \mathrm{O}\right)_{2} \mathrm{H}^{+}$and $\left(\mathrm{H}_{2} \mathrm{O}\right)_{3} \mathrm{H}^{+}$. The strength of these ion signals is not surprising given that the SCGD is sustained in direct contact with the liquid solution and that earlier studies have shown that large droplets ( $>10 \mu \mathrm{m}$ diameter) of the solution are ejected into the discharge during operation [7]. Such droplets should be rapidly vaporized within the discharge, where gas-kinetic temperatures are in excess of $3000 \mathrm{~K}$ [5], and in the heated inlet of the mass spectrometer, resulting in the formation of the smaller water clusters. Furthermore, given that the SCGD is sustained in air, ionized nitrogen and water molecules will also react quickly to form water clusters, similar to other plasmas sustained in the ambient air [22]. Expansion of the y-axis in Figure 2A reveals a variety of other reagent ions ( $c f$. Figure $2 \mathrm{~B}$ ). Though at lower relative abundance than the water clusters, $\mathrm{NO}^{+}, \mathrm{H}_{2} \mathrm{O}_{2}{ }^{+}, \mathrm{O}_{2}{ }^{+}, \mathrm{NO}_{2}{ }^{+}$, and $\mathrm{O}_{3}{ }^{+}$are also present in the mass spectrum, as observed with other atmospheric-pressure glow discharges [22-24]. The diverse range of reagent ions generated by the SCGD, including both proton donors (such as $\left(\mathrm{H}_{2} \mathrm{O}\right)_{\mathrm{n}} \mathrm{H}^{+}$) and charge-transfer reagents (e.g. $\mathrm{NO}^{+}, \mathrm{N}_{2} \mathrm{O}^{+}$, $\mathrm{O}_{2}{ }^{+}$, etc.), suggests that the SCGD should be useful for molecular mass spectrometry of varied polar and non-polar compounds [25].

Background ions produced by the SCGD were evaluated in the $m / z$ range of 50-500 Th, shown in Figure 2C. Though weak in comparison to the signal of $\left(\mathrm{H}_{2} \mathrm{O}\right)_{3} \mathrm{H}^{+}$a significant and structured background was observed over the range of $50-250 \mathrm{~m} / \mathrm{z}$. Though not verified experimentally, this complex, discrete background likely results from ions produced via thermal degradation of the polytetrafluoroethylene (PTFE) waste reservoir of the SCGD cell. This hypothesis is supported by earlier observations, where a similar background was observed in the mass spectrum produced from a helium atmospheric-pressure glow discharge housed in a PTFE cell body [26, 27]. Regardless of its source, this complex background is likely to be problematic for determination of elemental or low-mass molecular species, particularly if the SCGD were coupled to MS instruments that utilize low-resolution mass analyzers (e.g. quadrupolar ion trap, quadrupole, etc.). However, if the background does result from thermal degradation of the PTFE waste reservoir, it could be eliminated by use of a more thermally robust material (such as a ceramic), as was demonstrated in an earlier study by Shelley et al. [26].

In contrast to the complex positive-ion background, the negative-ion background of the SCGD is relatively simple. As shown in Figure 2D, this background consists only of nitrate ions $\left(\mathrm{NO}_{3}{ }^{-}\right)$and a

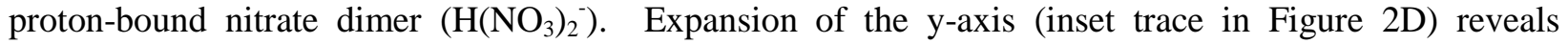
additional ions, though at far lower levels $(<0.5 \%$ abundance $)$ than $\mathrm{NO}_{3}^{-}$. The presence of strong signals from the negative reagent ion $\mathrm{NO}_{3}{ }^{-}$suggests that the SCGD might be useful for molecular analysis in the negative-ion mode. Furthermore, the low negative-ion background should lead to higher signal-to-noise $(\mathrm{S} / \mathrm{N})$ ratios and thereby better detection limits. Though negative ion detection was not explored in the present work beyond evaluation of the reagent ions, this possibility will be evaluated in future studies. 


\section{Elemental Analysis}

\section{Mass Spectra}

The ability to detect metal-containing species with SCGD-MS is demonstrated in Figure 3A, which shows the mass spectrum from a solution of 1-ppm $\mathrm{Cd}, \mathrm{Cu}, \mathrm{Ni}, \mathrm{Pb}$, and $\mathrm{U}\left(\mathrm{as}_{\mathrm{UO}_{2}}^{+}\right)$in $0.1 \mathrm{M}$ $\mathrm{HNO}_{3}$ introduced into the SCGD. From this solution, the SCGD produced both bare elemental ions and a variety of elemental ions bound with one or more adducts. Not surprisingly, the most common adduct was the addition of one or more water molecules to an elemental ion, though adducts of $\mathrm{O}_{1} \mathrm{NO}_{3}{ }^{-}$, and $\mathrm{OH}^{-}$ are also present. The presence of the many adduct ions in Figure 3A suggest that interpretation of elemental mass spectra could be difficult on low-resolution mass analyzers.

In-source collision-induced dissociation (CID) was explored as a means to reduce the clusters and adducts in the SCGD mass spectrum ( $c f$. Figure 3B). Here, a moderate potential $(40 \mathrm{~V})$ was applied between optics within the first stage of the mass spectrometer, which results in acceleration of all ions and more energetic collisions between ion pairs and with residual atmospheric gases. Conversion of the translational energy into vibrational energy results in dissociation of weaker chemical bonds. In the case of ions produced by the SCGD, in-source CID resulted in dissociation of most adducts. For $\mathrm{Cd}, \mathrm{Cu}$, and $\mathrm{Ni}$, only the bare metal ions were observed when in-source CID was utilized. For Pb, higher-order water adducts were eliminated, and only the bare elemental ions and adducts involving the addition of a single water were observed. For U, in-source CID resulted in the bare $\mathrm{UO}_{2}{ }^{+}$ion being most abundant, though water and hydroxide adducts were still present in the mass spectrum. Though in-source CID was used here because of its availability on the Orbitrap mass spectrometer, other methods of fragmentation would likely yield similar if not better results. For instance, here it was found that adjustment of the radiofrequency voltage on the S-lens of the Orbitrap also provided a means to decluster the ions.

Even though the SCGD is a relatively energetic ion source (average ion energy of $0.43 \mathrm{eV}$ ) [28], the resolution of the Orbitrap mass spectrometer is not degraded. Across the full mass range of Figures $3 \mathrm{~A}$ and $3 \mathrm{~B}$, resolution $(\mathrm{m} / \Delta \mathrm{m})$ was in excess of 100,000 , well within the range specified by the instrument manufacturer.

\section{Analytical Performance}

Detection limits and precision of SCGD-MS were measured for the strongest signal (bare elemental ion or adduct) for $\mathrm{Cu}, \mathrm{Cd}, \mathrm{Cs}, \mathrm{Pb}$, and $\mathrm{U}$ and the results are compiled in Table 1. Surprisingly, despite the large number of adducts formed for most elements, which result in dispersion of the signal across many $m / z$ channels, detection limits for SCGD-MS remained at levels useful for trace analyses, ranging from 0.1 to $4 \mathrm{ppb}$. Precision was found to be better than 16\% RSD at 1000-ppb concentration. These precision values are somewhat worse than those obtained with SCGD and optical emission spectrometry, where RSD values in the range of $0.5-3.5 \%$ are common [9-11, 29]. The comparatively poor precision here likely results from the method of ion sampling. No gas flows or electrostatic fields, apart from the vacuum pull of the first stage of the mass spectrometer and potential applied to the SCGD anode, were used to direct ions from the SCGD into the capillary of the mass spectrometer. Conceivably, future work towards optimization of both the source and sampling geometries could greatly enhance ion signals and precision.

Though in-source CID simplifies SCGD mass spectra ( $c f$. Figures 3A and 3B), it has little effect on quantitative analytical performance. Detection limits and precision of $\mathrm{Cu}, \mathrm{Cd}, \mathrm{Cs}, \mathrm{Pb}$, and $\mathrm{U}$ with in- 
source CID $(40 \mathrm{~V})$ are also included in Table 1. As before, the form of an element that produced the strongest ion signal was utilized for determining detection limits and precision-with CID, this was the bare elemental ion for all elements except $\mathrm{U}$, where $\mathrm{UO}_{2}{ }^{+}$was most abundant. With in-source CID, SCGD-MS detection limits ranged from 0.3 to $5 \mathrm{ppb}$; closely comparable to those obtained when CID was not used (0.1-4 ppb). Precision was generally similar also, from 5 to $11 \%$ RSD with in-source CID. Combined, these results suggest that any gains in signal achieved through dissociation of adducts are offset by ion losses when in-source CID is utilized. Though this finding suggests that CID is not necessary for acquisition of analytically useful data, the high resolution provided by the Orbitrap mass analyzer must be considered. If a lower-resolution mass analyzer were used, isobaric interferences from the many adduct series would be more problematic and could degrade detection limits. Under such conditions, analytical performance might improve appreciably when in-source CID or other broadband dissociation approach is utilized.

Detection limits of SCGD-MS are compared to those from SCGD-AES [9, 29], LS-APGD-MS [14], and ICP-MS [16, 30] in Table 2. In comparison to the results obtained via AES, the SCGD generally performs better with MS detection. Detection limits of SCGD-MS were between three and 60 times better than SCGD-AES for $\mathrm{Cd}, \mathrm{Cs}$, and $\mathrm{Pb}$, though the detection limit for $\mathrm{Cu}$ was identical with both detection methods. Similarly, SCGD-MS performs favorably in comparison to LS-APGD-MS for all elements investigated. Concentration-based detection limits for SCGD-MS are as much as 4.5 orders of magnitude better than those obtained with LS-APGD, though differences in sampling modes must be considered. In particular, LS-APGD detection limits were measured with 5- $\mu \mathrm{L}$ flow injections whereas continuous sample introduction (at a flow rate of $1.7 \mathrm{~mL} \mathrm{~min}^{-1}$ ) was utilized with the SCGD. In contrast, SCGD-MS detection limits are at least 20 times worse than those of ICP-MS ( $c f$. Table 2). Nevertheless, the results here are quite promising considering the early stages of SCGD investigation, and particularly differences in ion sampling. With ICP-MS, the plasma is positioned directly against the massspectrometer inlet, on-axis with the sampling orifice. By contrast, in the current design, ions from the SCGD are sampled radially at a distance $3 \mathrm{~mm}$ from the plasma. As a result, ion-transfer efficiency with the present SCGD geometry is likely quite poor in comparison to ICP-MS. Future work towards parametric optimization of the SCGD, combined with changes to source and inlet geometry that would promote efficient ion sampling are likely to enhance analytical performance.

Linear dynamic range for elemental analysis was also assessed. A working curve for Cs was measured over the range of $0.5-100,000 \mathrm{ppb}$, with standards introduced in half-decade concentration increments. It was found that Cs signal was linear from $0.5-10,000 \mathrm{ppb}(\log -\log$ plot slope of $0.97 \pm 0.03$, $\left.\mathrm{R}^{2}=0.99415\right)$, after which roll-off became apparent. If the Cs signal is assumed to maintain linearity to the 0.4-ppb detection limit, this corresponds to a linear working range of 4.4-orders of magnitude, similar to what is obtained for Cs with SCGD-AES (4.2 orders) [11]. Non-linearity beyond 10,000 ppb was found to be caused by condensation reactions that are preferential at high concentrations. Specifically, beyond a concentration of $10,000 \mathrm{ppb}, \mathrm{Cs}^{+}$signal leveled off, while signal from the nitrate-bound dimer, $\left(\mathrm{Cs}_{2} \mathrm{NO}_{3}\right)^{+}$, rose substantially. Dimer signal was apparent even at low concentrations (5 ppb), and is likely the reason the log-log slope is slightly less than one even over the linear portion of the Cs working curve. When the Cs curve was measured with in-source CID (40 V), roll-off still occurred at 10,000 ppb, despite the attenuation of the dimer signal. This finding suggests that though in-source CID is helpful in fragmenting clusters, it is not sufficient to recover the $\mathrm{Cs}^{+}$signal fully.

Potential for Speciation 
The distribution and types of adduct species formed from elemental salts are heavily influenced by solution composition. An example is shown in Figure 4, where 5-mM uranyl nitrate, $\mathrm{UO}_{2}\left(\mathrm{NO}_{3}\right)_{2}$, was introduced in a solution containing $1 \%(\mathrm{w} / \mathrm{w})$ acetic acid (Ac) and $0.1 \mathrm{M} \mathrm{HNO}_{3}$. Unlike the spectrum of Figure 3A, in which uranyl was introduced in $0.1 \mathrm{M} \mathrm{HNO}_{3}$, the presence of acetic acid resulted in a shift of the uranyl adducts to higher $\mathrm{m} / \mathrm{z}$ values. This shift results from the addition of acetate $\left(\mathrm{CH}_{3} \mathrm{COO}^{-}\right)$ anions to the uranyl cation ( $c f$. Figure 4). Furthermore, as acetic acid concentration was increased, signals from uranyl adducts bound to two acetate ions became more pronounced ( $c f$. Figure 5). Since the solution-phase form of the uranyl cation is dependent on $\mathrm{pH}$, especially in the presence of acetic acid [31], the observation of uranyl acetate adducts in the spectrum and their sensitivity to acetic acid concentration suggests that adducts present in the SCGD mass spectra might be useful for chemical speciation.

Unfortunately, there is rather poor correlation between the ion signals in Figure 4 and solutionphase molar ratios of uranyl acetate predicted from stability constants reported by Rao et al. [31]. At a $\mathrm{pH}$ of 1.0, as utilized here, nearly all of the uranyl $(\sim 92 \%)$ in solution should exist in the free cationic state $\left(\mathrm{UO}_{2}{ }^{2+}\right)$. Since ion signals corresponding to the free uranyl cation (such as $\mathrm{UO}_{2}{ }^{+},\left[\mathrm{UO}_{2}+\mathrm{OH}\right]^{+}$, $\left[\mathrm{UO}_{2}+\left(\mathrm{H}_{2} \mathrm{O}\right)_{n}+\mathrm{OH}\right]^{+}$, etc.) are greatly reduced by addition of acetic acid (cf. Figure 5), this suggests SCGD mass spectra are not representative of the solution-phase composition, just as was observed with the LS-APGD [17]. Similar difficulties were encountered in earlier studies on uranyl speciation by electrospray ionization MS [32], where it was postulated that large changes in solute concentration that occur during droplet desolvation result in the chemical equilibria being perturbed. Similar effects probably contribute to adduct distributions within the SCGD, as do gas-phase chemical reactions and clustering during trapping within the mass spectrometer.

Despite these difficulties, some adduct signals in SCGD-MS might be useful for speciation of elemental oxidation states. For instance, even when in-source CID was utilized, ion signals from uranium, introduced in solution as uranyl nitrate, retained the uranyl cation $\left(\mathrm{UO}_{2}{ }^{2+}\right)$ within the SCGD mass spectra and no elemental ion signal $\left(\mathrm{U}^{+}\right)$was observed ( $c f$. Figures $3 \mathrm{~A}$ and $3 \mathrm{~B}$ ). If other stable complex ions $\left(\mathrm{CrO}_{4}{ }^{2-}, \mathrm{MnO}_{4}^{-}, \mathrm{VO}^{3+}\right.$, etc. $)$ are similarly resistant to dissociation in the SCGD, the source could be used both to identify the oxidation state and to quantify these species separately from other chemical forms (e.g. $\mathrm{Cr}^{3+}, \mathrm{Mn}^{2+}, \mathrm{V}^{3+}$ ).

\section{Molecular Analysis}

\section{Molecular Mass Spectra from Solutions}

It was found that the SCGD can efficiently produce intact molecular ions from solution; three examples for 10- $\mu \mathrm{M}$ methyl salicylate, acetylacetone and isopropanol are shown in Figure 6. For both methyl salicylate and acetylacetone, the SCGD generated the protonated molecular ion $\left(\mathrm{MH}^{+}\right)$and fragments. Isopropanol exhibited only a single peak corresponding to the loss a hydrogen atom, likely a result of hydride abstraction facilitated by $\mathrm{NO}^{+}$, known to occur in chemical ionization sources [33]. No change in SCGD conditions or electrolyte composition was required to produce these ions. By comparison, to obtain molecular mass spectra with LS-AGPD, changes to the electrolyte composition are necessary. In the LS-APGD, introduction of analytes in acidic solution (e.g. $1 \mathrm{M} \mathrm{HNO}_{3}$ ) produces elemental mass spectra with comparatively weak, if any, molecular signals, whereas introduction of analytes in a 70:30 mixture of methanol and water results in molecular spectra similar to those observed here $[17,18]$. This feature, combined with the fact that the SCGD produces substantially more adducts in 
elemental analysis than does the LS-APGD [14, 34], suggests that the SCGD functions as a softer ionization source than the LS-APGD.

Earlier work with the SCGD as an ionization source for biomolecular mass spectrometry [20] revealed that fragmentation is tunable by adjustment of the discharge current. Similar results were observed here for small molecular species introduced within the liquid stream. A solution containing 10$\mu \mathrm{M}$ of a chemical warfare agent simulant, triethyl phosphate (TEP), was introduced into the SCGD in a carrier stream of $0.1 \mathrm{M} \mathrm{HCl}$. At an SCGD current of $35 \mathrm{~mA}$, the protonated molecular ion $\left(\mathrm{MH}^{+}\right)$of TEP at $\mathrm{m} / z 183.078$ is the most abundant species (cf. Figure 7A), though fragment ions, corresponding to successive losses of ethyl groups $\left(\mathrm{C}_{2} \mathrm{H}_{4}\right)$, were also detected. At the higher SCGD current of $70 \mathrm{~mA}$, the fragment ion at $m / z$ 98.984, corresponding to the loss of all three ethyl groups from TEP, became the base peak ( $c f$. Figure 7B). To assess differences in the "hardness" of ionization between the two current levels, survival yields (SY, a value directly related to hardness of ionization) [35] were calculated from the formula

$$
S Y=\frac{s_{p}}{S_{p}+\sum S_{f}}
$$

where $S_{p}$ and $S_{\mathrm{f}}$ are the mass spectral signals of the parent and fragment ions, respectively. Here, SY was $25.5 \%$ and $78.3 \%$ for currents of 70 and $35 \mathrm{~mA}$, respectively, indicating that the ionization process is "harder" (i.e. higher energy) at higher current (70-mA) and that fragmentation can be easily tuned by adjusting the source current. Interestingly, the trend in fragmentation with SCGD current here is reversed from that in the earlier study of the SCGD-based analysis of biopolymers, where low currents produced extensive fragmentation and high currents produced intact ions [20]. This difference hints that the mechanism of fragmentation of small molecular species (such as TEP) is different than that which leads to fragmentation of biopolymers, and that the mechanisms of ionization and fragmentation in the SCGD are complex.

Although detection limits for molecular species introduced in solution were not quantitatively determined, a rough estimate can be derived from the signal strength at concentrations used here. If the signal is assumed to drop linearly from $10-\mu \mathrm{M}$ concentration and the detection limit is conservatively estimated to occur at a signal level of $10^{3}$ counts, the protonated molecular ion of TEP, measured with an SCGD current of $35 \mathrm{~mA}$, would produce a detection limit of $1 \mathrm{nM}(0.2 \mathrm{ppb})$. This value is far lower than the short-term permissible limit of $10 \mathrm{ppb}$ in field drinking water imposed by the U.S. Army for the Gnerve agents that TEP simulates [36]. This result suggests that the SCGD-MS provides sufficient sensitivity for useful trace-level detection of small molecules.

\section{Ambient Desorption/Ionization}

The SCGD can also ionize ambient, molecular gases. For the proof-of-principle evaluation here, a bottle containing an organic solvent (either acetone or acetylacetone) was opened and placed on a laboratory bench approximately two meters from the SCGD and mass spectrometer inlet. Within seconds, the protonated molecular ion of both solvents was present at high abundance in the SCGD mass spectrum ( $c f$. Figures 8A and $\mathrm{B}$, respectively). Though this experiment is admittedly crude, Figure 8 clearly demonstrates the capability of the SCGD to ionize ambient vapors, and suggests that the source might be useful for gas-sampling applications (e.g. gas chromatography). However, for such applications to be reproducible and quantitative, the SCGD design would need to be modified. An SCGD format suitable for gas sampling was described by Pohl et al. [37], where a hollow anode was utilized to deliver a 
low flow of various gases directly into the discharge volume for optical emission experiments. A similar arrangement could be adapted easily to the SCGD-MS configuration.

The SCGD produces gas-phase ions from molecular species also introduced as solids. For this demonstration, the SCGD was sustained with a blank support solution $\left(0.1 \mathrm{M} \mathrm{HNO}_{3}\right)$ while a solid sample, deposited on the tip of a glass probe, was held between the SCGD and inlet of the mass spectrometer. Presumably, the high gas-kinetic temperature of the discharge heats the sample, resulting in thermal desorption of the deposited material. Interaction of desorbed material with reagent ions generated by the SCGD then results in ionization. When caffeine deposited on a glass probe was held within $2 \mathrm{~cm}$ of the SCGD, the protonated molecular ion $\left(\mathrm{MH}^{+}\right)$of caffeine at $m / z 195.087$ immediately appeared in the mass spectrum ( $c f$. Figure 9A). Though the protonated form was most abundant, oxidation products and a fragment that results from them (loss of $\mathrm{CO}$ ) were also present in the spectrum. Other small, polar molecules behaved similarly to caffeine when introduced as solids; Figures 9B and 9C show mass spectra for acetaminophen and urea, respectively. Just as with caffeine, $\mathrm{MH}^{+}$is highly abundant in both spectra and acetaminophen also exhibits fragments and oxidation products. Unlike caffeine or acetaminophen, urea produced a proton-bound dimer, while no fragments of urea were observed in the mass spectrum; though this finding is not surprising as many likely fragments of this ion would fall below the low-mass cutoff of the instrument.

Though protonation is a common ionization pathway for small polar molecules via ambient desorption/ionization with soft plasma-based sources [22, 38-41], detection of non-polar species with high boiling points is often more difficult. Figure 9D shows the SCGD mass spectrum obtained from the non-polar molecular p-quaterphenyl deposited on a glass probe. In contrast to the polar molecules, the radical molecular cation $\left(\mathrm{M}^{*+}\right)$ was the most abundant species, and a larger variety of adducts involving $\mathrm{NO}, \mathrm{NH}, \mathrm{O}, \mathrm{OH}$, and $\mathrm{H}_{2} \mathrm{O}$ were found in the spectrum. Presence of the radical molecular ion is indicative of a charge-transfer mechanism of ionization, and suggests that the reagent ions observed in Figure $2 \mathrm{~B}$ contribute to ionization, despite their low relative abundance compared to proton-donor regents ( $c f$. Figure 2A), as observed in other atmospheric-pressure glow discharges [25].

Surprisingly, sample and source positioning was not critical for ambient desorption/ionization via the SCGD. As long as the sample was held near (within 2-cm) the edge of the SCGD, mass spectral signals similar to those in Figures 9A-D were observed. This distance is larger than is required with other sources [18, 19, 22, 38-41], and hints at the possibility of non-proximate analysis of solids by the SCGD. Together, these findings indicate the flexibility of the SCGD as an ionization source for mass spectrometry.

\section{Conclusions}

Combined, these results suggest the versatility of the SCGD as an ion source for massspectrometric analysis of a wide variety of inorganic and organic analytes. Directly from aqueous solutions, the SCGD produced a variety of ions, ranging from bare elemental ions and adducts to intact organic molecules. Adducts in SCGD mass spectra might be useful for chemical speciation of stable complex ions such as $\mathrm{UO}_{2}{ }^{2+}$, which are resistant to dissociation in the SCGD. Furthermore, fragmentation of organic molecules introduced in solution is tunable merely by adjustment of SCGD current. Also, samples introduced as solids and gases are readily ionized by the SCGD, suggesting the source has applications in ambient ionization. Though much work remains to be done, this pilot study has identified several potential areas in which the SCGD might find MS application. 


\section{References}

[1] T. Cserfalvi, P. Mezei, P. Apai, Emission Studies on a Glow-Discharge in Atmospheric-Pressure Air Using Water as a Cathode, J Phys D Appl Phys, 26 (1993) 2184-2188.

[2] T. Cserfalvi, P. Mezei, Direct Solution Analysis by Glow-Discharge - Electrolyte-Cathode Discharge Spectrometry, J Anal Atom Spectrom, 9 (1994) 345-349.

[3] H.J. Kim, J.H. Lee, M.Y. Kim, T. Cserfalvi, P. Mezei, Development of open-air type electrolyte-ascathode glow discharge-atomic emission spectrometry for determination of trace metals in water, Spectrochimica Acta Part B: Atomic Spectroscopy, 55 (2000) 823-831.

[4] V.V. Yagov, M.L. Gentsina, Effect of Supporting Electrolyte Composition on the Intensity of Metal Lines in Electrolyte-Cathode Discharge Spectra, Journal of Analytical Chemistry, 59 (2004) 6470.

[5] M.R. Webb, F.J. Andrade, G. Gamez, R. McCrindle, G.M. Hieftje, Spectroscopic and electrical studies of a solution-cathode glow discharge, J Anal Atom Spectrom, 20 (2005) 1218-1225.

[6] M.R. Webb, F.J. Andrade, G.M. Hieftje, Use of electrolyte cathode glow discharge (ELCAD) for the analysis of complex mixtures, J Anal Atom Spectrom, 22 (2007) 766-774.

[7] A.J. Schwartz, S.J. Ray, E. Elish, A.P. Storey, A.A. Rubinshtein, G.C.Y. Chan, K.P. Pfeuffer, G.M. Hieftje, Visual observations of an atmospheric-pressure solution-cathode glow discharge, Talanta, 102 (2012) 26-33.

[8] A.J. Schwartz, Z. Wang, S.J. Ray, G.M. Hieftje, Universal Anion Detection by Replacement-Ion Chromatography with an Atmospheric-Pressure Solution-Cathode Glow Discharge Photometric Detector, Anal Chem, 85 (2013) 129-137.

[9] M.R. Webb, F.J. Andrade, G.M. Hieftje, Compact glow discharge for the elemental analysis of aqueous samples, Anal Chem, 79 (2007) 7899-7905.

[10] M.R. Webb, F.J. Andrade, G.M. Hieftje, High-throughput elemental analysis of small aqueous samples by emission spectrometry with a compact, atmospheric-pressure solution-cathode glow discharge, Anal Chem, 79 (2007) 7807-7812.

[11] A.J. Schwartz, S.J. Ray, G.M. Hieftje, Evaluation of Interference Filters for Spectral Discrimination in Solution-Cathode Glow Discharge Optical Emission Spectrometry, J Anal Atom Spectrom, (2016).

[12] R.K. Marcus, W.C. Davis, An Atmospheric Pressure Glow Discharge Optical Emission Source for the Direct Sampling of Liquid Media, Anal Chem, 73 (2001) 2903-2910.

[13] W.C. Davis, R.K. Marcus, Role of powering geometries and sheath gas composition on operation characteristics and the optical emission in the liquid sampling-atmospheric pressure glow discharge, Spectrochimica Acta Part B: Atomic Spectroscopy, 57 (2002) 1473-1486.

[14] C.D. Quarles, A.J. Carado, C.J. Barinaga, D.W. Koppenaal, R.K. Marcus, Liquid samplingatmospheric pressure glow discharge (LS-APGD) ionization source for elemental mass spectrometry: preliminary parametric evaluation and figures of merit, Analytical and Bioanalytical Chemistry, 402 (2012) 261-268.

[15] L.X. Zhang, B.T. Manard, S.K. Kappel, R.K. Marcus, Evaluation of the operating parameters of the liquid sampling-atmospheric pressure glow discharge (LS-APGD) ionization source for elemental mass spectrometry, Anal Bioanal Chem, 406 (2014) 7497-7509.

[16] S.J. Hill, Fishers, A., Foulkes, M., Inductively Coupled Plasma and Its Applications, 2nd ed. ed., Blackwell Publishing Ltd.: Oxford2007.

[17] L.X. Zhang, B.T. Manard, B.A. Powell, R.K. Marcus, Preliminary Assessment of Potential for Metal-Ligand Speciation in Aqueous Solution via the Liquid Sampling-Atmospheric Pressure Glow Discharge (LS-APGD) Ionization Source: Uranyl Acetate, Anal Chem, 87 (2015) 72187225 . 
[18] L.X. Zhang, R.K. Marcus, Mass spectra of diverse organic species utilizing the liquid samplingatmospheric pressure glow discharge (LS-APGD) microplasma ionization source, J Anal Atom Spectrom, 31 (2016) 145-151.

[19] R.K. Marcus, C.Q. Burdette, B.T. Manard, L.X. Zhang, Ambient desorption/ionization mass spectrometry using a liquid sampling-atmospheric glow discharge (LS-APGD) ionization source, Analytical and Bioanalytical Chemistry, 405 (2013) 8171-8184.

[20] A.J. Schwartz, J.T. Shelley, C.L. Walton, K.L. Williams, G.M. Hieftje, Atmospheric-Pressure Ionization and Fragmentation of Peptides by Solution-Cathode Glow Discharge, Chemical Science, (2016).

[21] G. Wells, H. Prest, C.R. Russ, Signal, Noise, and Detection Limits in Mass Spectrometry, Application Note, Agilent Technologies, 2011

[22] F.J. Andrade, J.T. Shelley, W.C. Wetzel, M.R. Webb, G. Gamez, S.J. Ray, G.M. Hieftje, Atmospheric Pressure Chemical Ionization Source. 1. Ionization of Compounds in the Gas Phase, Anal Chem, 80 (2008) 2646-2653.

[23] Y. Cao, J.-H. Choi, B.-M. Haas, M. Okumura, Spectroscopic sudies of the intracluster hydration reaction of NO2+, The Journal of Physical Chemistry, 98 (1994) 12176-12185.

[24] C.A. Vasko, D.X. Liu, E.M. van Veldhuizen, F. Iza, P.J. Bruggeman, Hydrogen Peroxide Production in an Atmospheric Pressure RF Glow Discharge: Comparison of Models and Experiments, Plasma Chemistry and Plasma Processing, 34 (2014) 1081-1099.

[25] S.P. Badal, S.D. Michalak, G.C.Y. Chan, Y. You, J.T. Shelley, Tunable Ionization Modes of a Flowing Atmospheric-Pressure Afterglow (FAPA) Ambient Ionization Source, Anal Chem, 88 (2016) 3494-3503.

[26] J.T. Shelley, J.S. Wiley, G.M. Hieftje, Ultrasensitive Ambient Mass Spectrometric Analysis with a Pin-to-Capillary Flowing Atmospheric-Pressure Afterglow Source, Anal Chem, 83 (2011) 57415748.

[27] G.D. Schilling, J.T. Shelley, J.H. Barnes, R.P. Sperline, M.B. Denton, C.J. Barinaga, D.W. Koppenaal, G.M. Hieftje, Detection of positive and negative ions from a flowing atmospheric pressure afterglow using a mattauch-herzog mass spectrograph equipped with a faraday-strip array detector, Journal of the American Society for Mass Spectrometry, 21 (2010) 97-103.

[28] M.R. Webb, G.C.Y. Chan, F.J. Andrade, G. Gamez, G.M. Hieftje, Spectroscopic characterization of ion and electron populations in a solution-cathode glow discharge, J Anal Atom Spectrom, 21 (2006) 525-530.

[29] A.J. Schwartz, S.J. Ray, G.M. Hieftje, Automatable On-line Generation of Calibration Curves and Standard Additions in Solution-Cathode Glow Discharge Optical Emission Spectrometry, Spectrochimica Acta Part B: Atomic Spectroscopy, 105 (2015) 77-83.

[30] K.J. Stetzenbach, M. Amano, D.K. Kreamer, V.F. Hodge, Testing the Limits of ICP-MS: Determination of Trace Elements in Ground Water at the Part-Per-Trillion Level, Ground Water, 32 (1994) 976-985.

[31] J. Jiang, L. Rao, P.D. Bernardo, P. Zanonato, A. Bismondo, Complexation of uranium(vi) with acetate at variable temperatures, Journal of the Chemical Society, Dalton Transactions, (2002) 1832-1838.

[32] S. Pasilis, Á. Somogyi, K. Herrmann, J.E. Pemberton, Ions Generated from Uranyl Nitrate Solutions by Electrospray Ionization (ESI) and Detected with Fourier Transform Ion-Cyclotron Resonance (FT-ICR) Mass Spectrometry, Journal of the American Society for Mass Spectrometry, 17 (2006) 230-240.

[33] A.G. Harrison, Chemical ionization mass spectrometry, 2nd ed., CRC Press, Boca Raton, Fla., 1992.

[34] R.K. Marcus, C.D. Quarles, C.J. Barinaga, A.J. Carado, D.W. Koppenaal, Liquid SamplingAtmospheric Pressure Glow Discharge Ionization Source for Elemental Mass Spectrometry, Anal Chem, 83 (2011) 2425-2429. 
[35] M. Nefliu, J.N. Smith, A. Venter, R.G. Cooks, Internal energy distributions in desorption electrospray ionization (DESI), Journal of the American Society for Mass Spectrometry, 19 (2008) 420-427.

[36] U. Green, J.H. Kremer, M. Zillmer, C. Moldaenke, Detection of chemical threat agents in drinking water by an early warning real-time biomonitor, Environmental Toxicology, 18 (2003) 368-374.

[37] P. Jamróz, W. Żyrnicki, P. Pohl, The effect of a miniature argon flow rate on the spectral characteristics of a direct current atmospheric pressure glow micro-discharge between an argon microjet and a small sized flowing liquid cathode, Spectrochimica Acta Part B: Atomic Spectroscopy, 73 (2012) 26-34.

[38] R.B. Cody, J.A. Laramée, H.D. Durst, Versatile New Ion Source for the Analysis of Materials in Open Air under Ambient Conditions, Anal Chem, 77 (2005) 2297-2302.

[39] F.J. Andrade, J.T. Shelley, W.C. Wetzel, M.R. Webb, G. Gamez, S.J. Ray, G.M. Hieftje, Atmospheric Pressure Chemical Ionization Source. 2. Desorption-Ionization for the Direct Analysis of Solid Compounds, Anal Chem, 80 (2008) 2654-2663.

[40] J.D. Harper, N.A. Charipar, C.C. Mulligan, X. Zhang, R.G. Cooks, Z. Ouyang, Low-Temperature Plasma Probe for Ambient Desorption Ionization, Anal Chem, 80 (2008) 9097-9104.

[41] M.-Z. Huang, C.-H. Yuan, S.-C. Cheng, Y.-T. Cho, J. Shiea, Ambient Ionization Mass Spectrometry, Annual Review of Analytical Chemistry, 3 (2010) 43-65. 


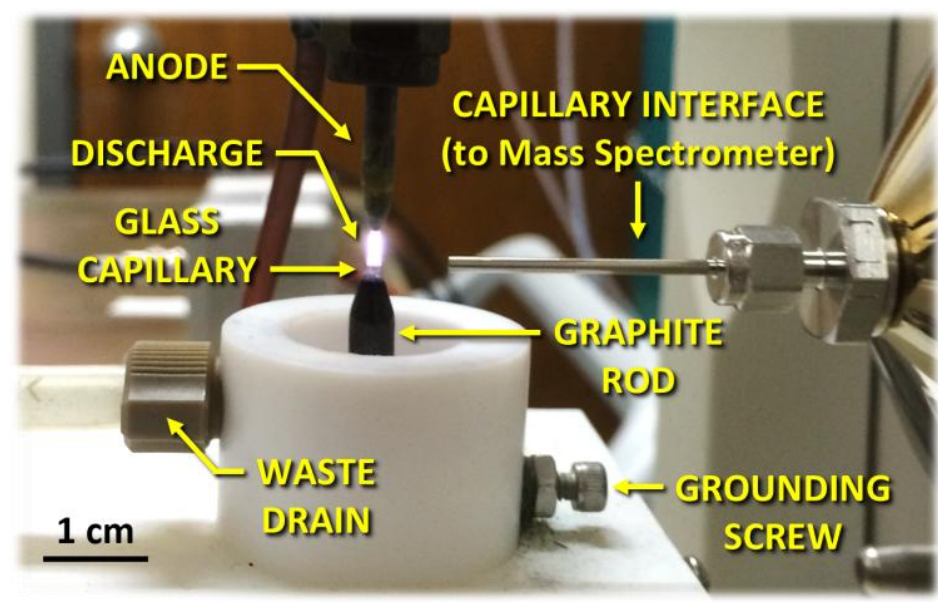

Figure 1. Photograph of the SCGD cell and capillary interface used for mass-spectrometry experiments. 

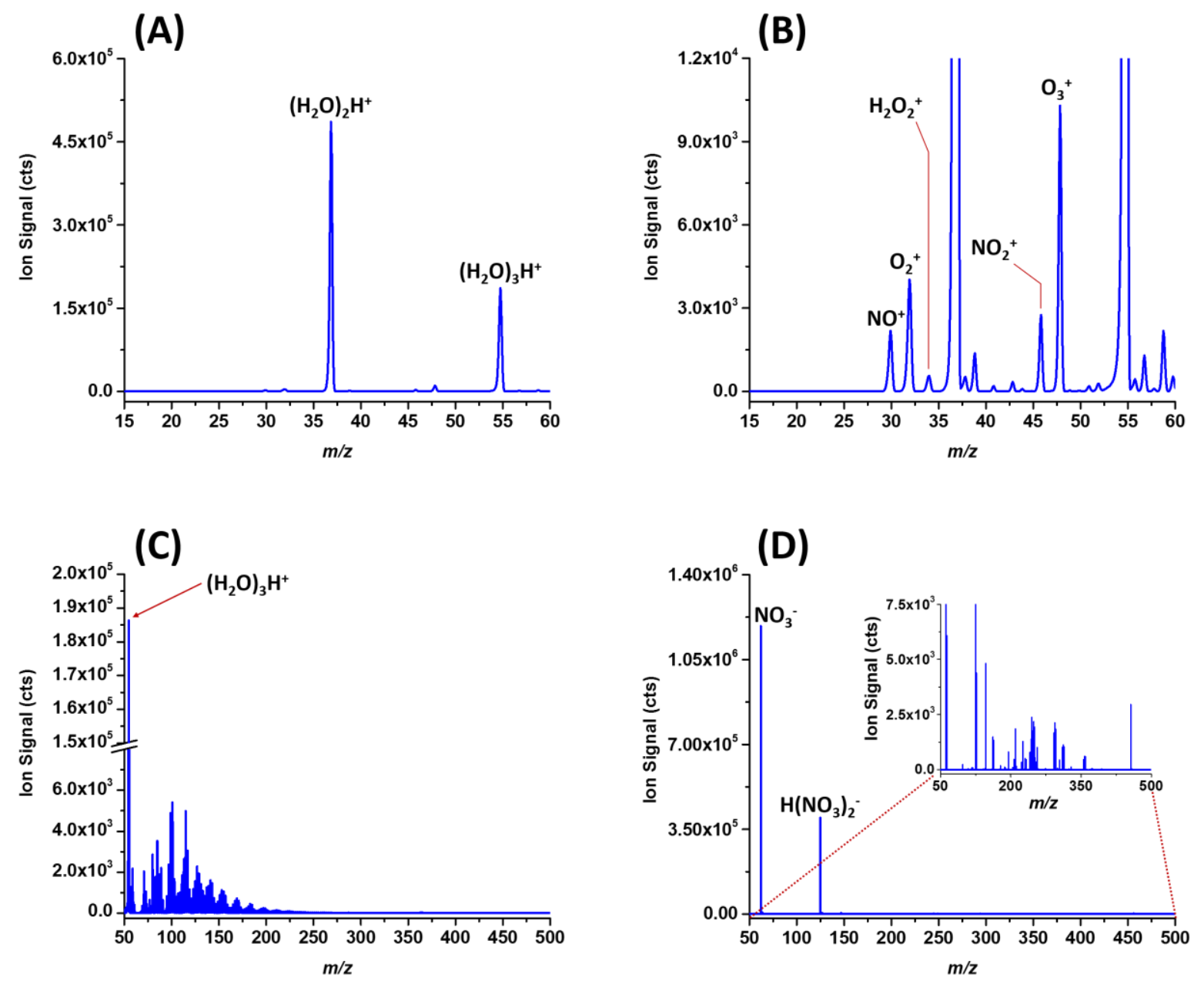

Figure 2. Mass spectra of reagent and background ions formed by the SCGD during introduction of a 0.1 $\mathrm{M} \mathrm{HNO}_{3}$ blank solution. Spectra (A) and (B) display the low- $m / z$ reagent ions, while (C) and (D) show positive- $(\mathrm{C})$ and negative- (D) ion background. 
Figure 3. SCGD mass spectra of a solution of 1-ppm $\mathrm{Cd}, \mathrm{Cu}, \mathrm{Ni}, \mathrm{Pb}$ and $\mathrm{U}$ in $0.1 \mathrm{M} \mathrm{HNO}_{3}$ without (A) and with (B) first-stage collision-induced dissociation.

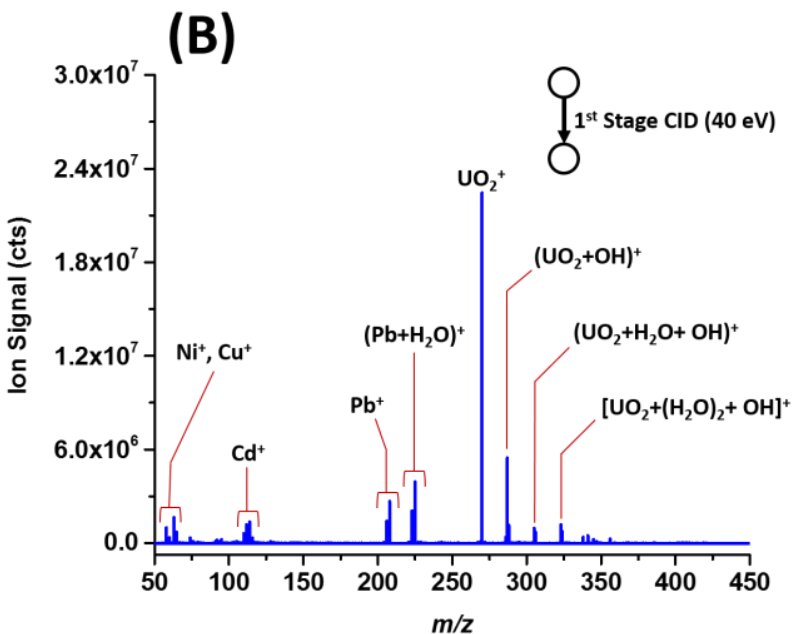


Figure 4. SCGD-mass spectrum of $5-\mathrm{mM} \mathrm{UO}_{2}{ }^{2+}$ solution in $0.1 \mathrm{M} \mathrm{HNO}_{3}$ with $1 \%(\mathrm{w} / \mathrm{w})$ acetic acid. Note differences in $\mathrm{UO}_{2}{ }^{2+}$ adducts from spectrum in Figure $3 \mathrm{~A}$. 


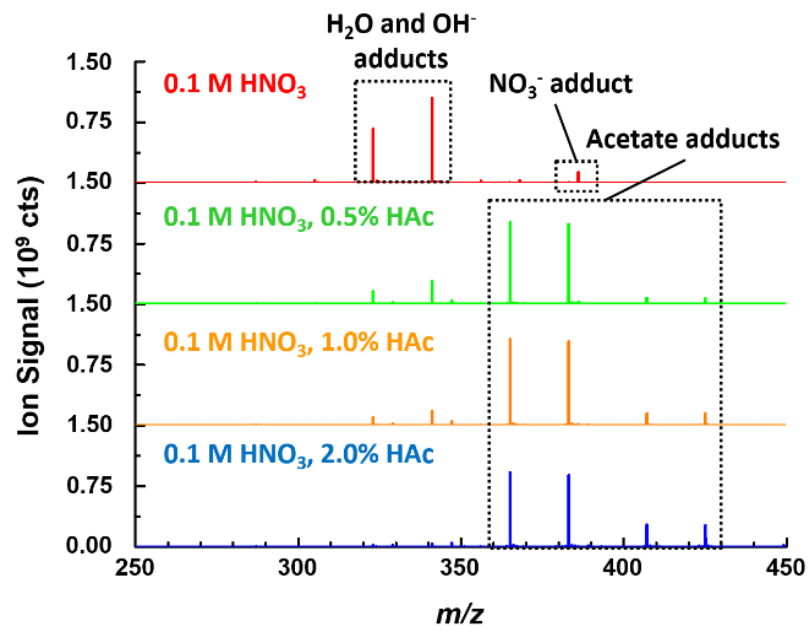

Figure 5. SCGD mass spectra demonstrate the change in adduct distribution of $5-\mathrm{mM} \mathrm{UO}_{2}{ }^{2+}$ in $0.1 \mathrm{M}$ $\mathrm{HNO}_{3}$ with varied amounts of acetic acid. 

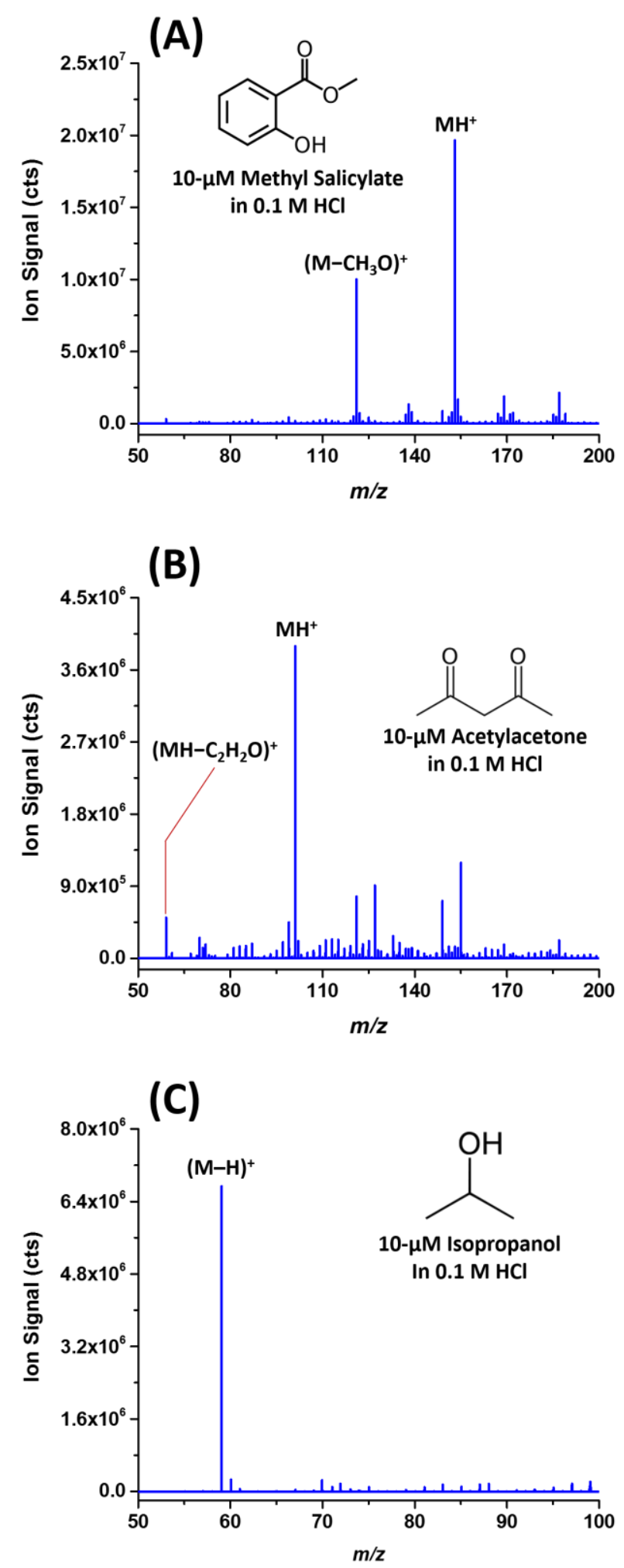

Figure 6. Mass spectra of $10-\mu \mathrm{M}$ methyl salicylate (A), acetylacetone (B), and isopropanol (C) introduced in $0.1 \mathrm{M} \mathrm{HCl}$ and obtained with an SCGD current of $70 \mathrm{~mA}$. 
Figure 7. Mass spectra of $10-\mu \mathrm{M}$ triethyl phosphate in $0.1 \mathrm{M} \mathrm{HCl}$ at SCGD currents of 70- (A) and 35(B) $\mathrm{mA}$. Note the differences in fragment-ion abundances. 

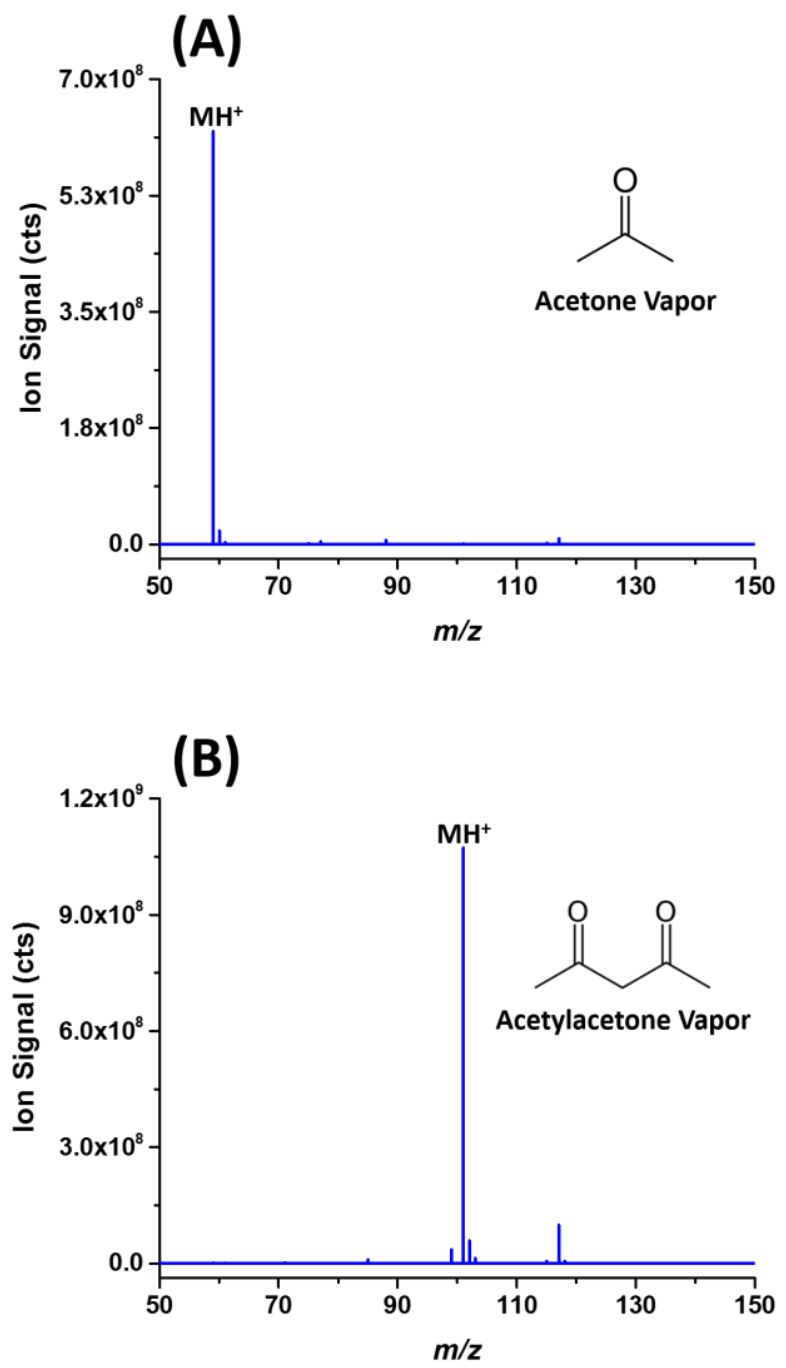

Figure 8. Ionization and detection of acetone (A) and acetylacetone (B) vapor by SCGD-MS. 
(A)

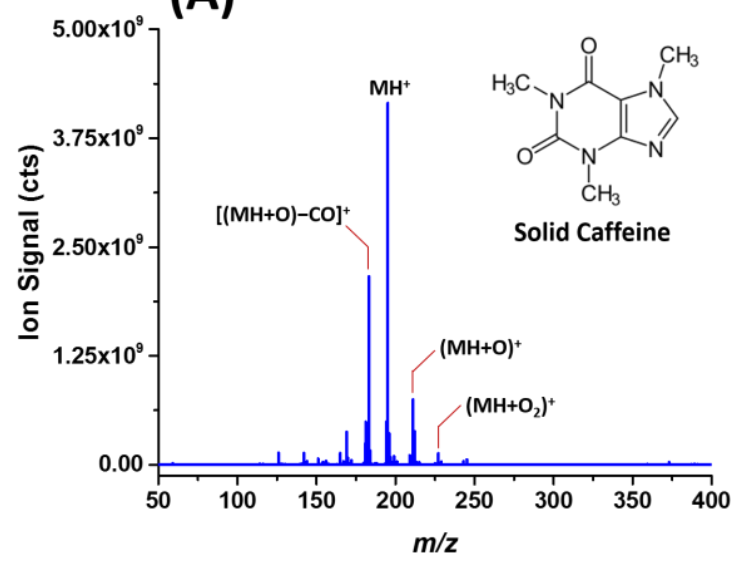

(C)

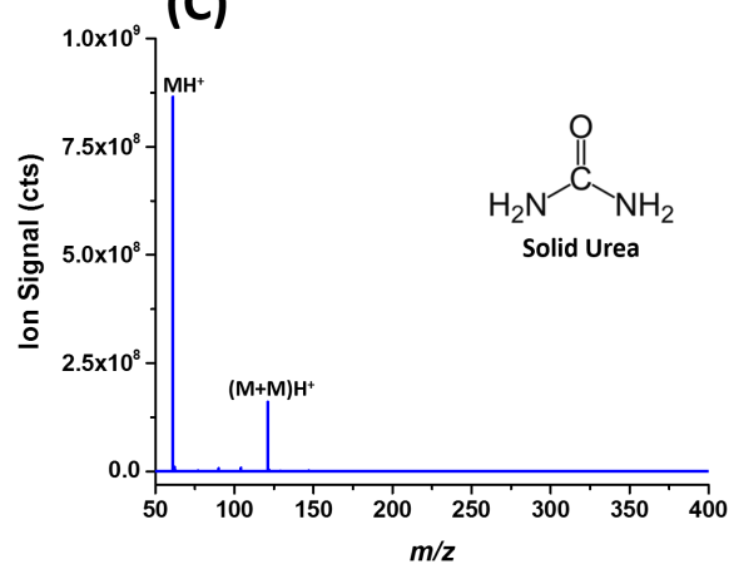

(B)
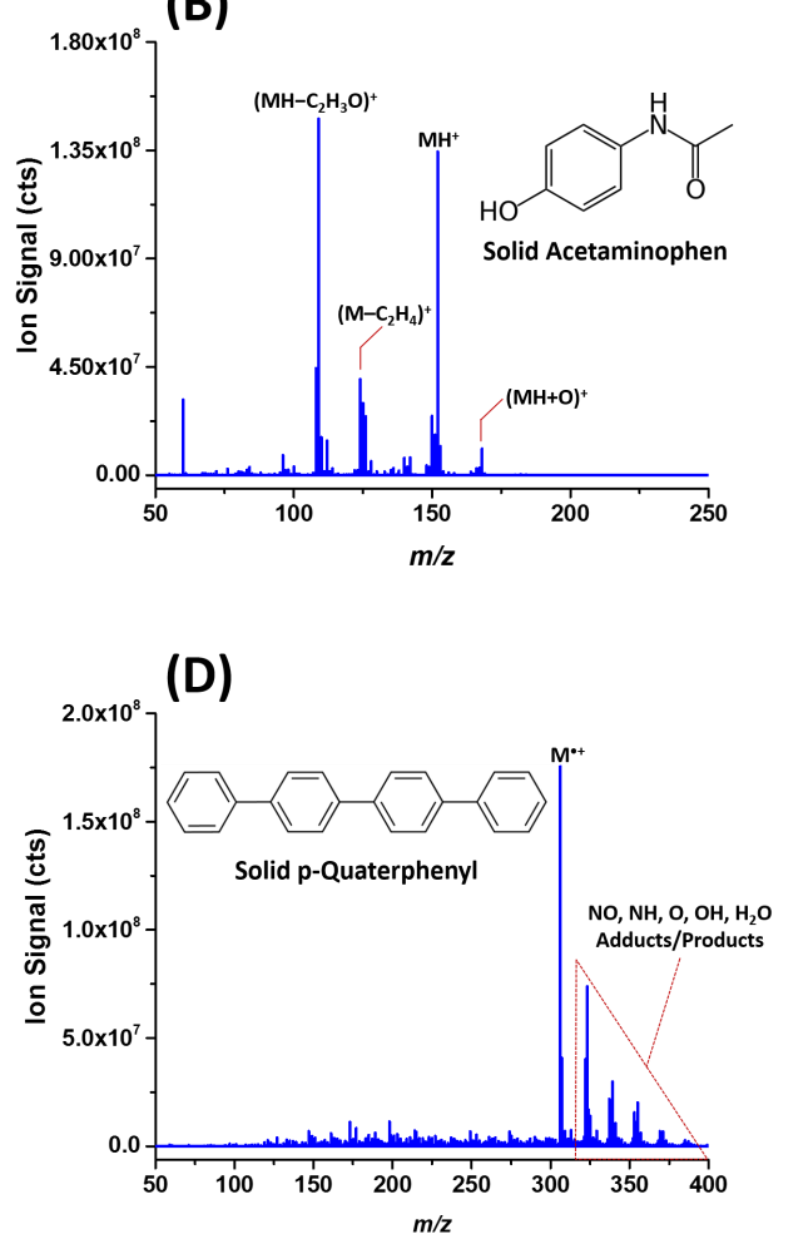

Figure 9. Single-scan ambient desorption/ionization mass spectra of solid caffeine (A), acetaminophen (B), urea (C) and p-quaterphenyl (D), deposited individually on a glass probe. The SCGD was operated at a current of $70 \mathrm{~mA}$ with a blank support solution of $0.1 \mathrm{M} \mathrm{HNO}_{3}$. 
Table 1. Detection limits and precision of SCGD-MS for elemental species with and without in-source CID.

\begin{tabular}{cccc|ccc}
\hline \multirow{2}{*}{ Element } & \multicolumn{2}{c|}{ SCGD, without in-source CID } & \multicolumn{3}{c}{ SCGD, with in-source CID } \\
Detection & $\begin{array}{c}\text { Form } \\
\text { Measured }\end{array}$ & $\begin{array}{c}\text { RSD }^{\dagger} \\
\text { Limit (ppb) }\end{array}$ & $\begin{array}{c}\text { Form } \\
(\boldsymbol{\%})\end{array}$ & $\begin{array}{c}\text { Detection } \\
\text { Measured }\end{array}$ & $\begin{array}{c}\text { RSD }^{\dagger} \\
\text { Limit (ppb) }\end{array}$ & $(\boldsymbol{\%})$ \\
\hline $\mathrm{Cd}$ & {$\left[\mathrm{CdNO}_{3}+2\left(\mathrm{H}_{2} \mathrm{O}\right)\right]^{+}$} & 0.1 & 10 & $\mathrm{Cd}^{+}$ & 0.4 & 10 \\
$\mathrm{Cs}$ & $\mathrm{Cs}^{+}$ & 0.4 & 11 & $\mathrm{Cs}^{+}$ & 0.3 & 5 \\
$\mathrm{Cu}$ & {$\left[\mathrm{Cu}+\left(\mathrm{H}_{2} \mathrm{O}\right)_{2}\right]^{+}$} & 4 & 16 & $\mathrm{Cu}^{+}$ & 5 & 11 \\
$\mathrm{~Pb}$ & {$\left[\mathrm{~Pb}+\mathrm{H}_{2} \mathrm{O}\right]^{+}$} & 0.2 & 7 & $\mathrm{~Pb}^{+}$ & 0.6 & 9 \\
$\mathrm{U}$ & {$\left[\mathrm{UO}_{2}+\mathrm{OH}+\mathrm{H}_{2} \mathrm{O}\right]^{+}$} & 0.8 & 8 & $\left(\mathrm{UO}_{2}\right)^{+}$ & 0.4 & 7 \\
\hline
\end{tabular}

"Detection limits were determined from the sum of all isotopic signals of the form specified.

${ }^{\dagger}$ Measured at a concentration of $1000 \mathrm{ppb}$ with 5 repetitions (9.4-s integration). 
Table 2. Comparison of SCGD-MS detection limits with those obtained by SCGD-AES, LS-APGD-MS, and ICP-MS.

\begin{tabular}{ccccc}
\hline \multirow{2}{*}{ Element } & \multicolumn{4}{c}{ Limit of Detection (ppb) } \\
& SCGD-MS & $\begin{array}{c}\text { SCGD-AES } \\
{[\mathbf{9 , 2 9 ]}}\end{array}$ & $\begin{array}{c}\text { LS-APGD-MS } \\
{[\mathbf{1 4}]}\end{array}$ & $\begin{array}{c}\text { ICP-MS } \\
{[\mathbf{1 6}, \mathbf{3 0}]}\end{array}$ \\
\hline $\mathrm{Cd}$ & 0.1 & 6 & 600 & 0.005 \\
$\mathrm{Cs}$ & 0.4 & 1 & 50 & 0.0005 \\
$\mathrm{Cu}$ & 4 & 4 & 2200 & 0.005 \\
$\mathrm{~Pb}$ & 0.2 & 2 & 20 & 0.008 \\
$\mathrm{U}$ & 0.8 & - & - & 0.001 \\
\hline
\end{tabular}




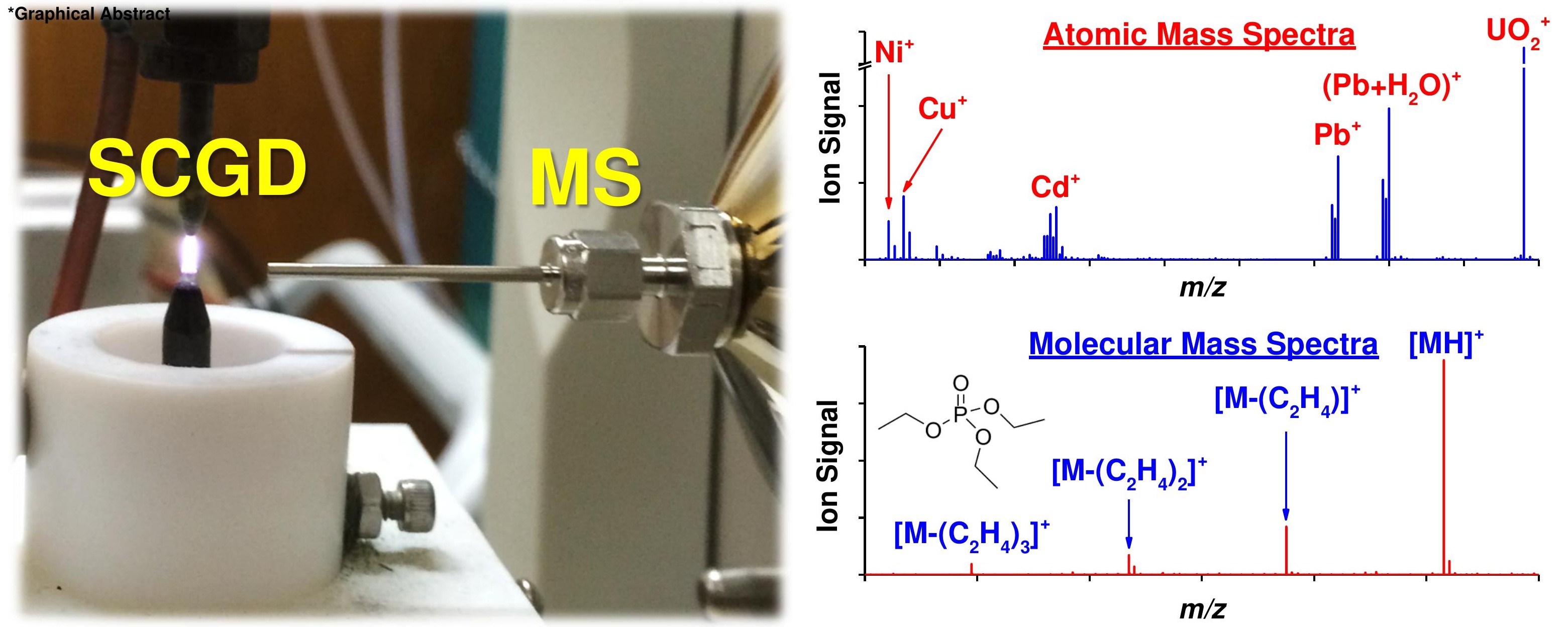

